\title{
Impact of design-parameters on the optical performance of a high- power adaptive mirror
}

\author{
Wouter D. Koek*, David Nijkerk, Jeroen A. Smeltink, Teun C. van den Dool, \\ Erwin J. van Zwet, Gregor E. van Baars \\ TNO, Stieltjesweg 1, 2628CK, Delft, The Netherlands
}

\begin{abstract}
TNO is developing a High Power Adaptive Mirror (HPAM) to be used in the $\mathrm{CO}_{2}$ laser beam path of an Extreme UltraViolet (EUV) light source for next-generation lithography. In this paper we report on a developed methodology, and the necessary simulation tools, to assess the performance and associated sensitivities of this deformable mirror. Our analyses show that, given the current limited insight concerning the process window of EUV generation, the HPAM module should have an actuator pitch of $\leq 4 \mathrm{~mm}$.

Furthermore we have modelled the sensitivity of performance with respect to dimpling and actuator noise. For example, for a deformable mirror with an actuator pitch of $4 \mathrm{~mm}$, and if the associated performance impact is to be limited to smaller than $5 \%$, the actuator noise should be smaller than $45 \mathrm{~nm}(\mathrm{rms})$. Our tools assist in the detailed design process by assessing the performance impact of various design choices, including for example those that affect the shape and spectral content of the influence function.
\end{abstract}

Keywords: adaptive optics, deformable mirror, actuator pitch, dimpling, top hat, EUV source, conversion efficiency

\section{INTRODUCTION}

In order to sustain Moore's law ${ }^{1}$, which says that computing power will double every two years, it is imperative that the semi-conductor industry makes the transition to extreme ultra-violet (EUV) lithography for high volume manufacturing $(\mathrm{HVM})^{2}$. The radiation sources needed for HVM EUV lithography rely on laser-produced plasmas (LPP) to produce the required EUV radiation ${ }^{3-5}$. In such a source a target (typically either disc-shaped liquid tin or a cloud of vaporized tin droplets) ${ }^{6}$ is illuminated with a focused high-power pulsed $\mathrm{CO}_{2}$ laser (wavelength $10.6 \mu \mathrm{m}$ ).

LPP sources typically operate in a double-pulse scheme in which a first pulse, the so-called pre-pulse (PP), serves to form the target from a droplet of liquid tin. A second pulse, the so-called main-pulse (MP), then illuminates the target to generate the EUV. The energy of the main-pulse is typically much higher than the energy of the pre-pulse. This paper focuses on the design of an adaptive mirror to be used for control of the spatial profile of the MP spot.

The PP and MP lasers, and subsequent optical components, have typically been designed to generate a Gaussian $\left(\sim \mathrm{TEM}_{00}\right)$ beam profile and thus also a Gaussian far-field (i.e. focused) profile. In practice however, there is always a trade-off between laser output power and beam quality, leading to beam profiles that are not perfectly Gaussian. A consequence is that the MP spot profile varies as a function of axial, through-focus, position (and is also seen to differ from system to system). It is important to point out that LPP systems may be aligned such that the tin target, that has a $\sim 300 \mu \mathrm{m}$ diameter $^{6}$, is (slightly) outside the MP beam waist, as this has been shown to lead to more efficient generation of EUV radiation ${ }^{7}$. One benefit of an adaptive optical system is that it helps ensuring the beam profile to be well-defined for various positions around the beam waist, and reproduces well between different systems.

A deformable mirror not only helps to improve stability and reproducibility, more importantly, it is expected that a tophat MP spot will result in a higher EUV conversion efficiency than when using a Gaussian MP spot ${ }^{8,9}$. At TNO we have developed a high power adaptive mirror (HPAM) that allows to generate a top-hat MP spot in and around the beam waist position in the focal region. This deformable mirror both enables experimental research into the impact of MP spot shape onto EUV generation, and allows for the anticipated subsequent integration of an actively controlled MP spot shape into HVM EUV sources.

*wouter.koek@tno.nl; phone +31-88-866-8002

High-Power Laser Materials Processing: Applications, Diagnostics, and Systems VI, edited by Stefan Kaierle,

Stefan W. Heinemann, Proc. of SPIE Vol. 10097, 1009704 · () 2017 SPIE

CCC code: $0277-786 X / 17 / \$ 18 \cdot$ doi: $10.1117 / 12.2249739$ 


\section{BACKGROUND INFORMATION}

In this chapter we will first present relevant background information. We will briefly present the requirements of the deformable mirror, and discuss the various design-parameters taken into account in this study. Next we discuss the methodology and metrics that were used to analyze the performance of the deformable mirror. In the next chapter we will then present the results of how the various design-parameters affect the optical performance of an HPAM-equipped system.

\subsection{Requirements}

The main operational parameters for the design of the HPAM module are:

$\begin{array}{ll}\text { Laser power: } & <50 \mathrm{~kW} \\ \text { Laser pulse energy: } & <500 \mathrm{~mJ} \\ \text { Laser wavelength: } & 10.6 \mu \mathrm{m} \\ \text { Laser beam diameter: } & 32 \mathrm{~mm}(\mathrm{D} 4 \sigma) \\ \text { Clear optical aperture: } & 50 \mathrm{~mm} \\ \text { Top hat diameter: } & 300 \mu \mathrm{m}\end{array}$

\subsection{Design-parameters}

Prior to starting the opto-mechanical design of the deformable mirror it is important to set proper requirements on several design parameters.

As schematically shown in Figure 1 a deformable mirror consist of a (thin) reflective membrane, commonly referred to as facesheet, on which a spatial profile can be induced by controlling the length of individual actuators which reside in back of the facesheet. Note that depending on the particular type of implementation (e.g. stacked versus in-plane actuator) the actuators might not have a direct connection to the fixed world ${ }^{10}$.

The pitch and the inter-actuator stroke are important input parameters for the opto-mechanical performance and design of the mirror. Generally a smaller pitch leads to increased complexity (and may exclude the ability to use a certain type of actuator), but is expected to result in better optical performance. The design of a deformable mirror is always a balancing act between realizing a mirror that will do what it is meant for, yet without being overly complicated (and thus expensive). Since the cost of a deformable mirror is roughly assumed to be linear with the number of actuators, the actuator pitch is an important parameter in this trade-off.

The aim of the current study is to enable the detailed opto-mechanical design of the HPAM module by determining the performance related sensitivities with respect to actuator pitch (Chapter 3.1.1) and actuator noise (Chapter 3.2). Furthermore, the developed models also indicate the required inter-actuator stroke and allow the opto-mechanical designers to test the performance impact of the influence function (Chapter 3.3).

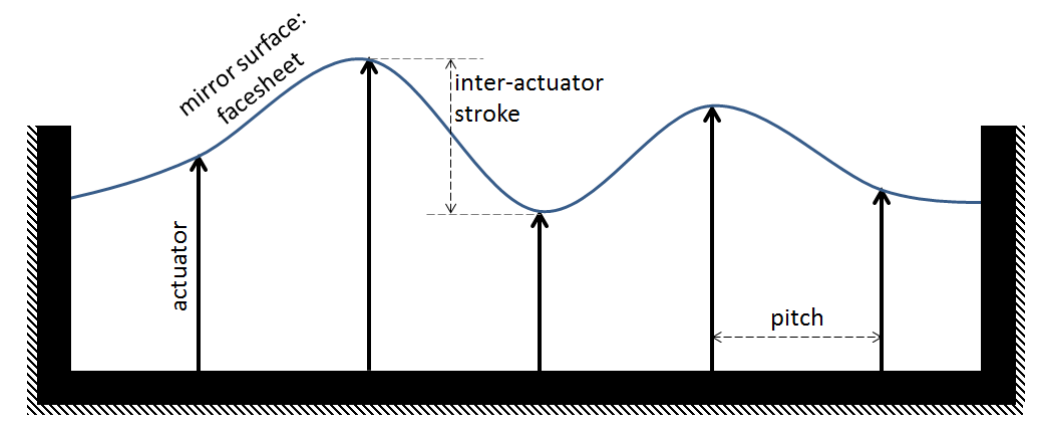

Figure 1. Schematic representation of a deformable mirror. A flexible reflective surface (the facesheet) is bent into shape by an array of actuators. The spatial frequencies that can be introduced in the mirror (and thus the incoming wavefront) are limited by the pitch between neighboring actuators, whereas the magnitude of the phase shift that can be induced at these high frequencies is determined by the inter-actuator stroke. 


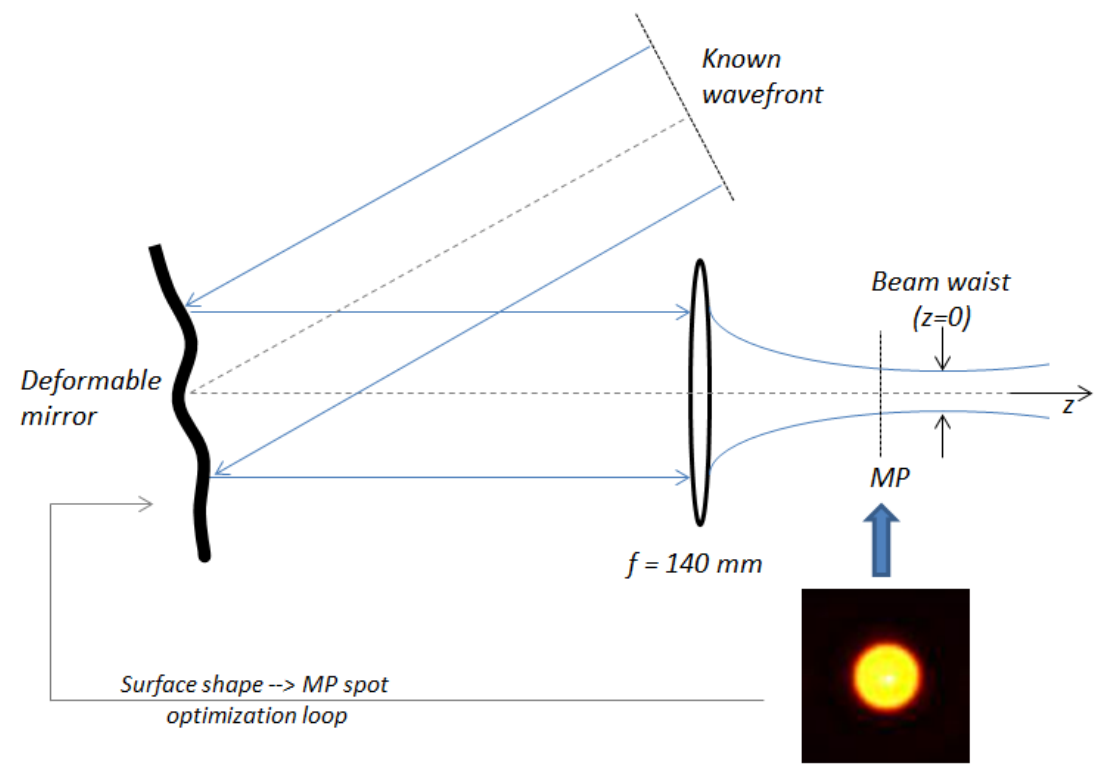

Figure 2. Schematic representation of the methodology used in this numerical study.

\subsection{Methodology}

Figure 2 shows an extremely simplified schematic representation of the optical system in an LPP source. Please note that only those parts of the system that are relevant for this study have been schematically shown. We will now use Figure 2 to give a step-by-step explanation of our analysis methodology:

- We start our analysis given a known wavefront at a location in the beam path some distance from the deformable mirror. This wavefront has been obtained by measurement (typically using a Phasics SID4 wavefront sensor).

- The wavefront in the plane of the deformable mirror is now numerically determined (using Angular Spectrum Propagation $)^{11}$.

- Since the influence functions (the resulting surface deformation when driving an individual actuator) of the deformable mirror are known (these have been measured and/or modelled), and the deformable mirror behaves as a linear system, all practically realizable surface shapes can be modelled. Typically, an analysis initially assumes a flat state of the deformable mirror (which will then later be iteratively optimized).

- For a certain mirror shape, and thus changes in optical path length, the localized phase change that the mirror imparts on the incoming beam can easily be determined. Given a certain shape of the deformable mirror the reflected wavefront can thus be determined; this wavefront is then propagated to the focusing optics.

- If there is system specific aberration data of the focusing optics available this can be applied to the wavefront.

- Knowing the optical properties of the focusing optics, the irradiance profile is determined for a number of (through-focus) $z$-planes.

- Typically the goal of an analysis is to optimize the MP spot profile in a particular $z$-plane between the focusing optics and the beam waist. This is achieved by using a gradient descent optimization algorithm on the actuator control voltages (and thus the surface shape of the deformable mirror) and iteratively propagating between the deformable mirror and the MP plane until the MP spot meets the required performance metric (see Chapter 2.4)

By repeating the above described procedure for varying simulation parameters (such as actuator pitch, noise levels, or by using wavefront data from a different system), the performance related sensitivities can be readily determined. 


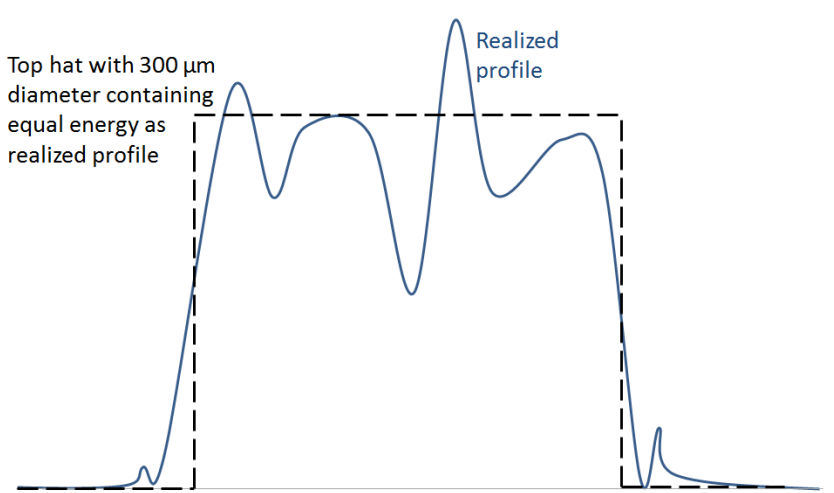

(a)

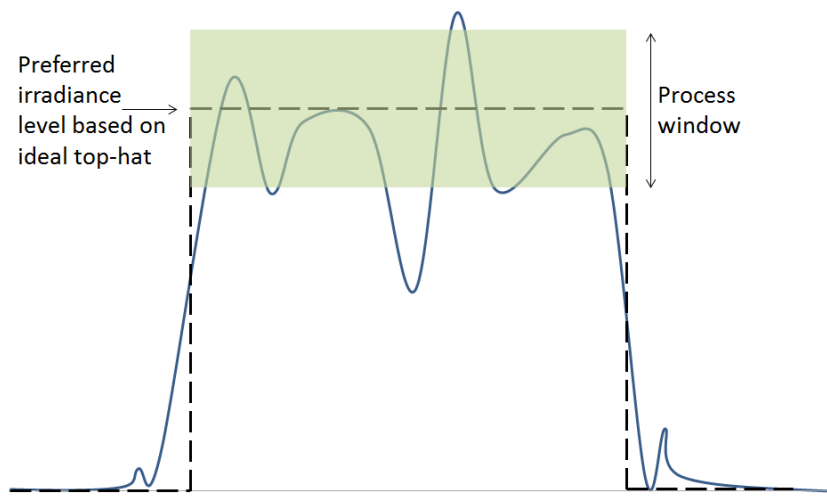

(b)

Figure 3. Metrics to assess the performance of the deformable mirror. (a) Root of Sum of Squares metric; (b) Uniformity metric.

\subsection{Metrics}

The goal of the HPAM module is to generate a well-defined (top-hat) MP spot. We will now define proper metrics to assess its performance.

The most obvious metric is to compare the realized MP profile with an ideal top hat. Figure 3(a) shows a cross section of both a realized profile $R$ and an ideal top hat $T$ (having a $300 \mu \mathrm{m}$ diameter and containing equal energy as $R$ ).

We now define the Root of Sum of Squares (RSS) performance of $\mathrm{R}$ as:

$$
R S S=\sqrt{\sum_{x, y}\left(R_{x, y}-T_{x, y}\right)^{2}},
$$

where $x, y$ refers to the spatial coordinates of the MP profile.

It may however be the case that it is not strictly required to obtain full uniformity of the realized profile, but rather the irradiances should lie within a certain process window (see Figure 3(b)).

We thus also define the ' $X \%$ uniformity' metric $\left(U_{X \%}\right)$ as the surface fraction of $T$ (within a $300 \mu \mathrm{m}$ diameter) where $R$ lies within $X \%$ of $T$ :

$$
U_{X \%}=\frac{\sum_{x, y}\left\{\left[R_{x, y} \geq(100 \%-X \%) T_{x, y}\right] \&\left[R_{x, y} \leq(100 \%+X \%) T_{x, y}\right] \&\left[T_{x, y}>0\right]\right\}}{\sum_{x, y}\left[T_{x, y}>0\right]},
$$

where the square brackets ' []' refer to a Boolean operation and \& denotes a logical AND.

It is important to realize that although the presence of a process window may be expected from a physical point of view, the width of this window is currently unknown. In fact, the availability of an HPAM module would greatly facilitate an experimental effort to determine the process window.

Given this current uncertainty with respect to the process window, in our analyses we therefore use RSS as the leading metric, although various uniformity metrics $\left(U_{10 \%}, U_{25 \%}, U_{50 \%}\right)$ will also be determined for reference.

Although apparent from their definitions, we would like to point out that a better profile results in a lower $R S S$ yet in a higher $U_{X \%}$. 


\section{RESULTS AND DISCUSSION}

In this chapter we will present and discuss the results of various analyses that were performed throughout this study.

\subsubsection{Actuator pitch}

Following the procedure that was explained before, the surface shape of the deformable mirror was optimized with respect to RSS for a set of actuator pitches. This analysis assumes a Gaussian-shaped influence function with a full-width half-maximum width (FWHM) of 1.2 times the actuator pitch.

Figure 4 shows the pitch-dependent performance of the deformable mirror, whereas Figure 5 and Figure 6 show the resulting MP spot profiles for various actuator pitches. The blue stars in Figure 4 indicate the performance without a deformable mirror, however this should not be interpreted as 'poor performance' of the system as-is. It should be remembered that the system has been designed to result in a Gaussian profile, and as can be seen in Figure 5(a) the resulting MP profile is indeed fairly Gaussian. Note that, although Figures 4-6 show data for a single laser system, many different laser systems have been analyzed. These other systems show similar behavior, thereby leading to the same conclusions.

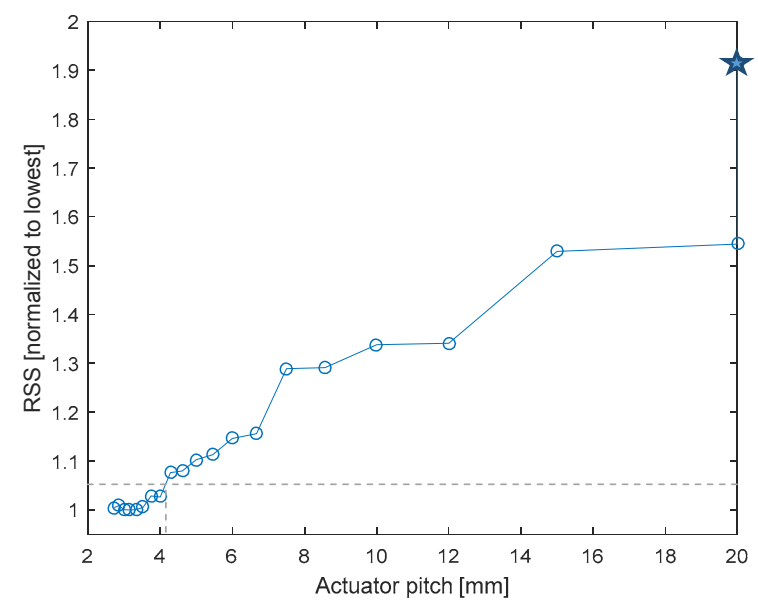

(a)

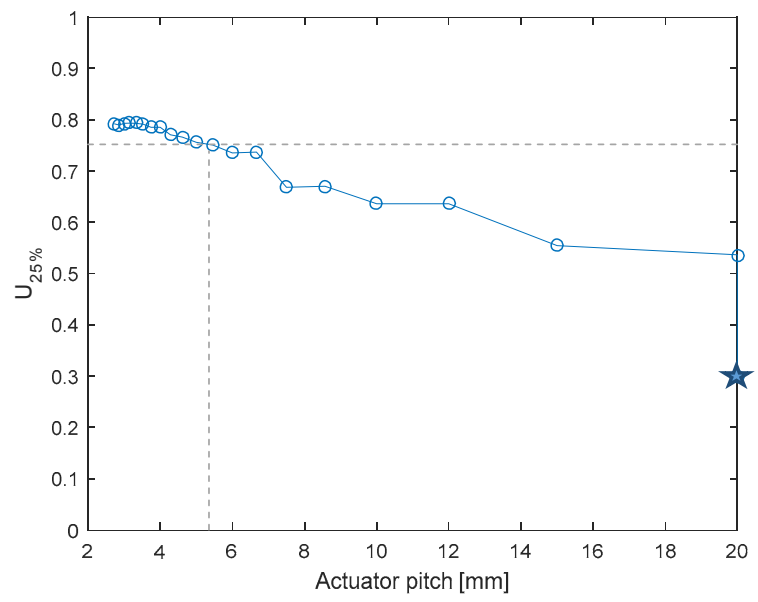

(c)

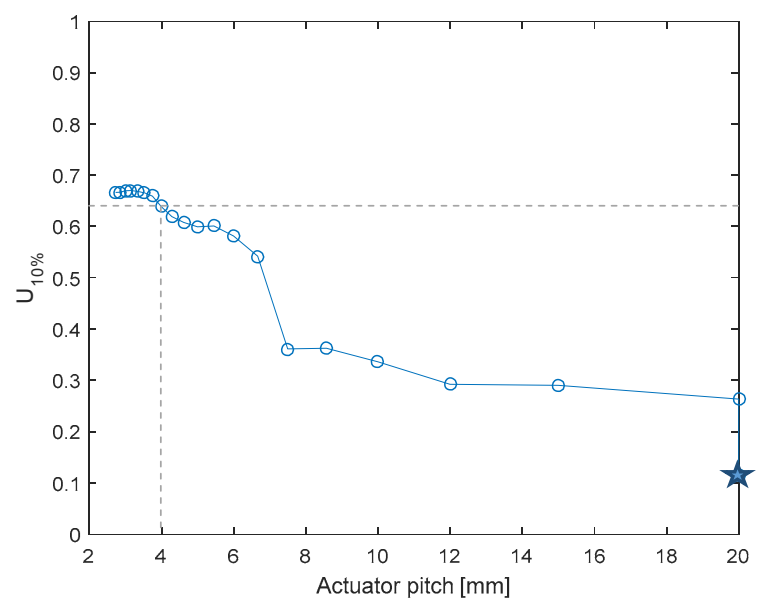

(b)

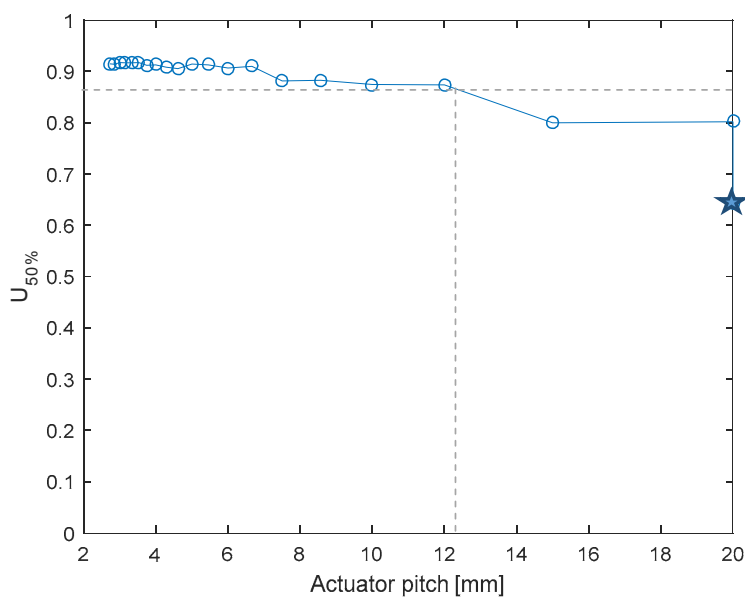

(d)

Figure 4. Influence of actuator pitch on various performance metrics of the deformable mirror. The blue star in each graph indicates the performance without a deformable mirror. The dashed lines indicate the actuator pitch where the performance is decreased by $5 \%$ with respect to optimal performance. 


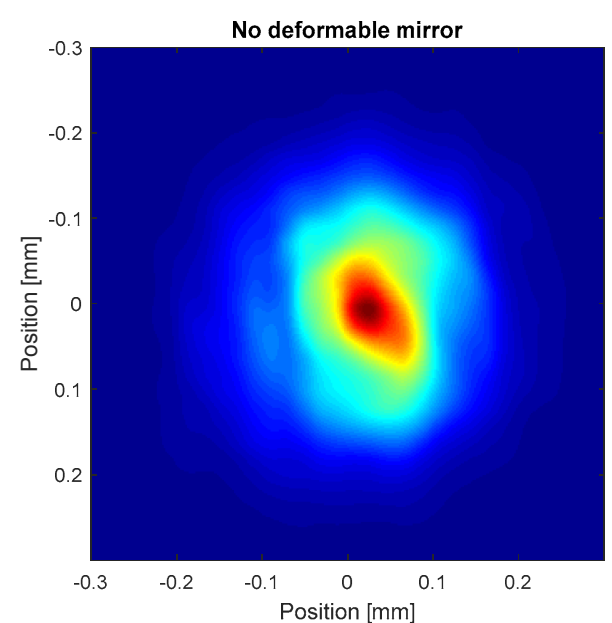

(a)

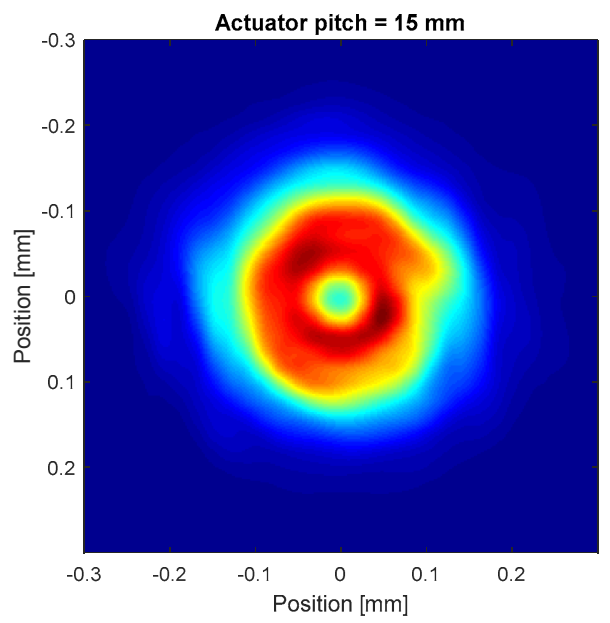

(c)

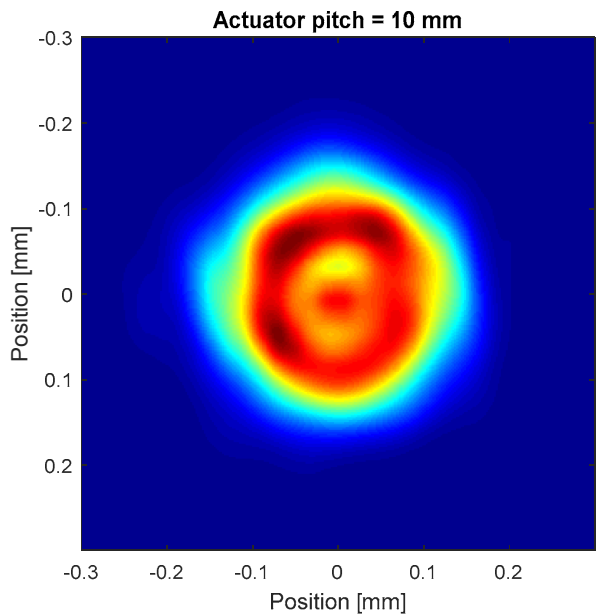

(e)

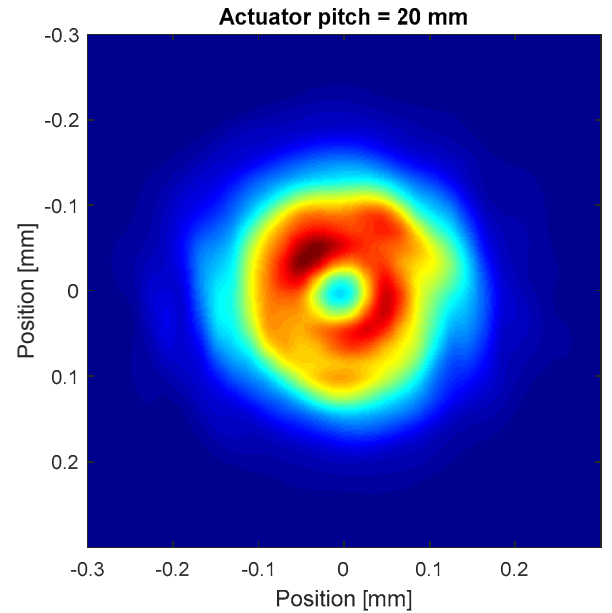

(b)

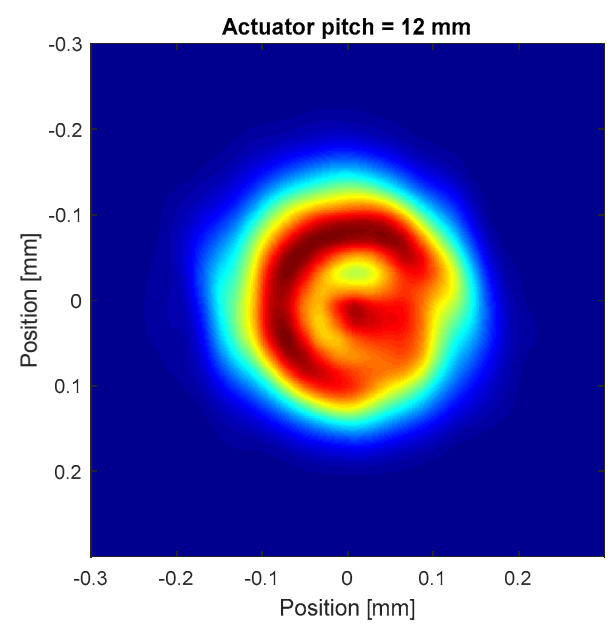

(d)

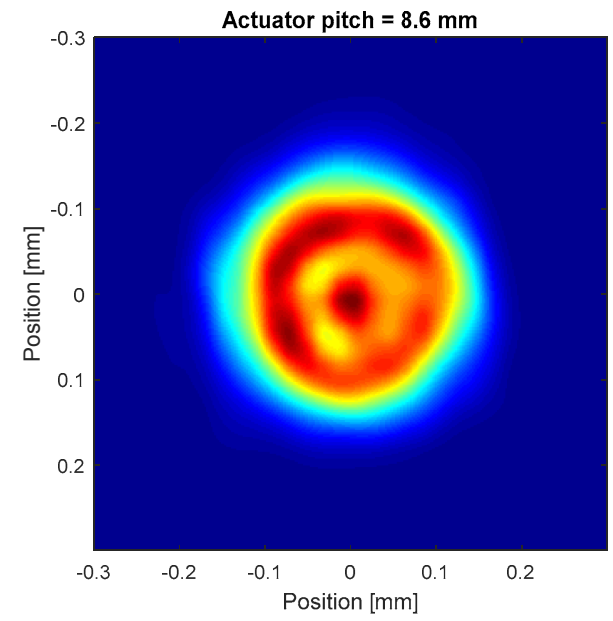

(f)

Figure 5. MP spot profile (a) without deformable mirror (only focus has been optimized), and (b-f) for a deformable mirror with various actuator pitches. 


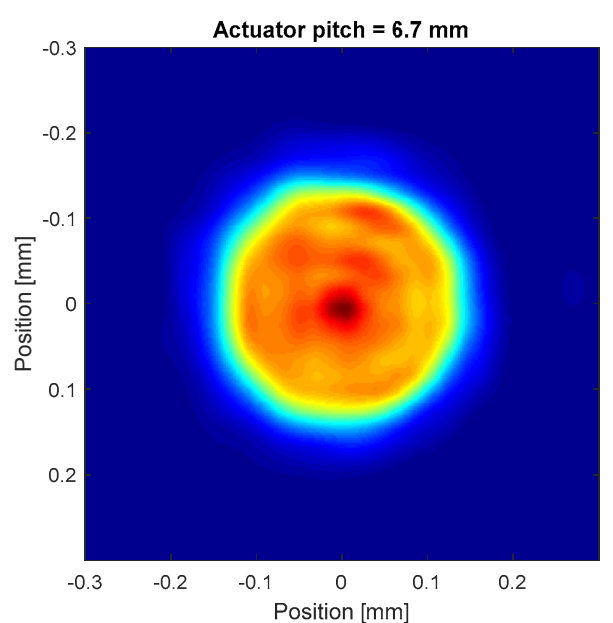

(a)

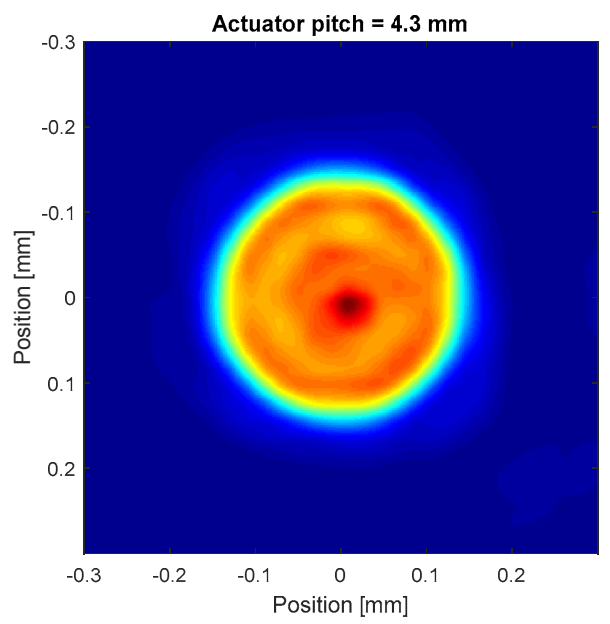

(c)

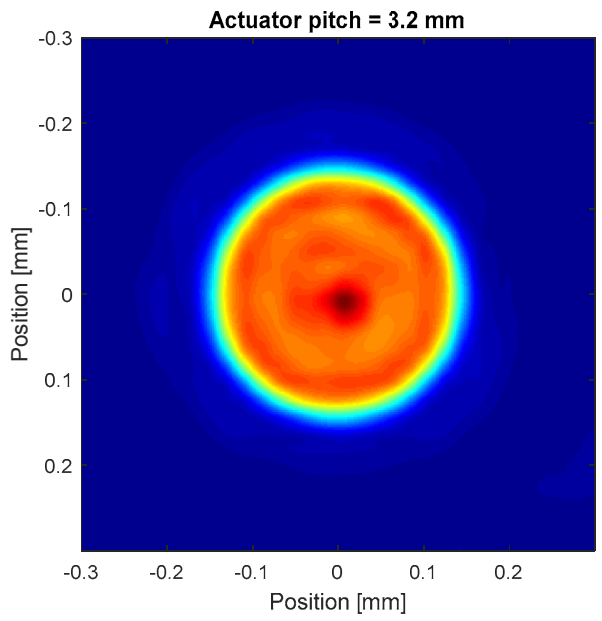

(e)

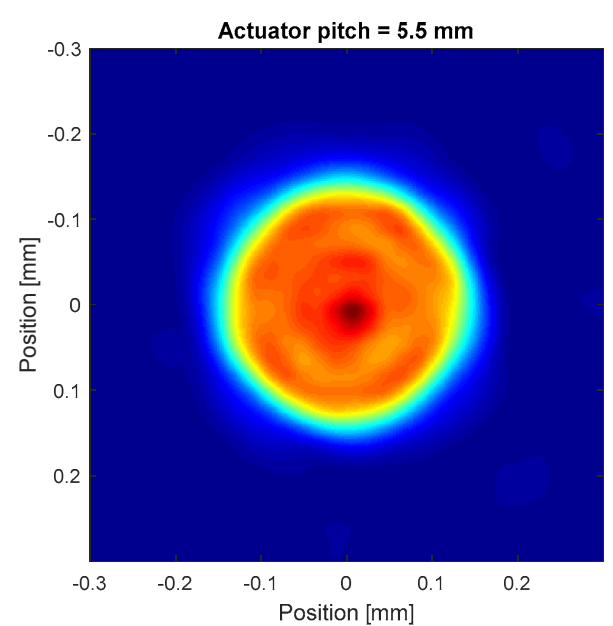

(b)

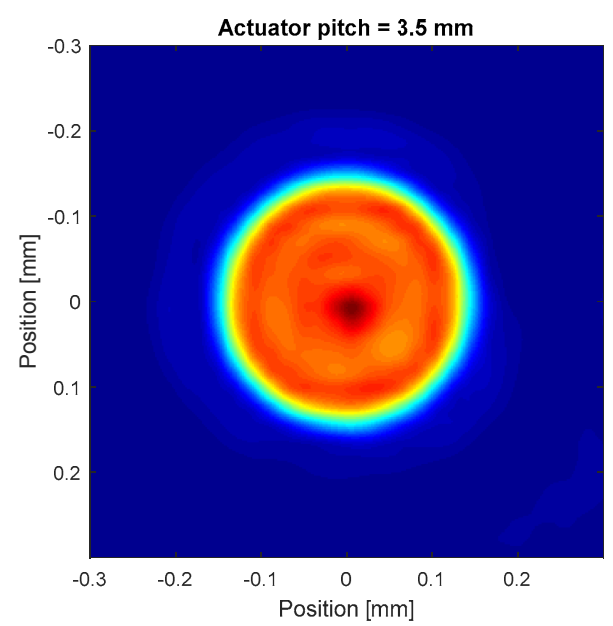

(d)

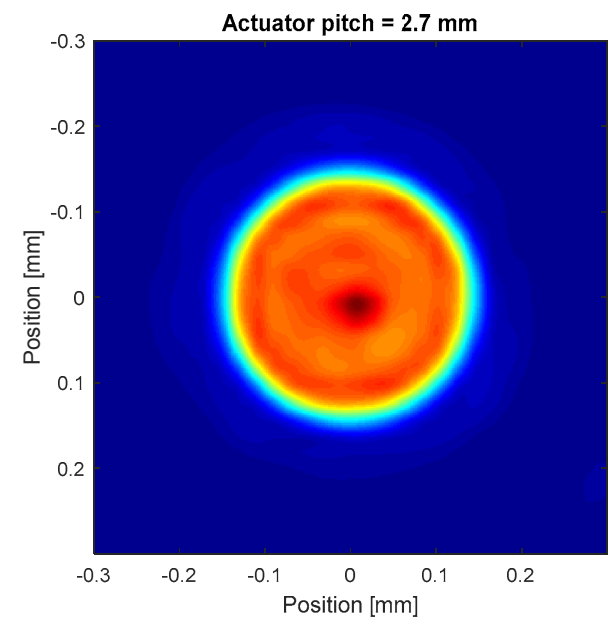

(f)

Figure 6. MP spot profile for a deformable mirror with various actuator pitches (continuation of the series in Figure 5). 
Figure 4 clearly shows that the ability to realize a top hat MP profile is greatly improved by equipping the system with a deformable mirror.

Figure 4 also indicates that more knowledge about the process window of EUV generation may actually impact the requirements of the HPAM module. When considering the $R S S$ and $U_{10 \%}$ metrics, having an actuator pitch equal to (or below) $4 \mathrm{~mm}$ is needed maintain a performance level that is within $5 \%$ of optimal performance. However, when judging the system by the $U_{50 \%}$ metric, an actuator pitch of $12 \mathrm{~mm}$ suffices to maintain such a performance level. As explained earlier, due to the limited knowledge concerning the process window, for now we recommend to base the requirements of the HPAM module on $R S S$, and to set the required actuator pitch at $\leq 4 \mathrm{~mm}$.

The various curves in Figure 4 do not show a simple smooth relation between the actuator pitch and the performance metric, but there are a number of performance plateaus. We have seen this behavior also for the other laser systems that we have analyzed. These plateaus imply that a certain critical spatial frequency must be enforced upon the deformable mirror to enable 'next level' performance.

Such transitions in RSS are typically clearly observed at $20 \mathrm{~mm}$ (coming from a system without deformable mirror), between 12-15 mm and between 7-8 mm. As can be seen in Figure 5 and Figure 6 these transition regimes correspond to having the required spatial frequency content to:

Around $20 \mathrm{~mm}$ : Form a donut spot (and correct astigmatism should this be present in the system)

Around $15 \mathrm{~mm}$ : Fill up the hole in the donut with a central peak, and improve edge steepness

Around $7 \mathrm{~mm}$ : Smoothen central part of spot

For actuator pitches $<7 \mathrm{~mm}$ a gradual improvement is observed as the steepness of the edges is increased, and the top hat uniformity improves.

\subsubsection{Dimpling}

The actuator pitch not only determines the spatial frequencies that can intentionally be induced on the facesheet, but also affects how sensitive the design will be to unwanted (and uncorrectable) inter-actuator surface deformations.

As schematically shown in Figure 7, even when all actuators are set to the same height, thermal effects may lead to local surface deformations ('dimpling') ${ }^{12-14}$. Since the maximum spatial frequency that can be corrected by an actuator grid with pitch $P$ is equal to $1 /(2 \cdot P)$, yet the dimpling induces a spatial frequency of $1 / P$, these thermally-induced surface deformations tend to be uncorrectable.

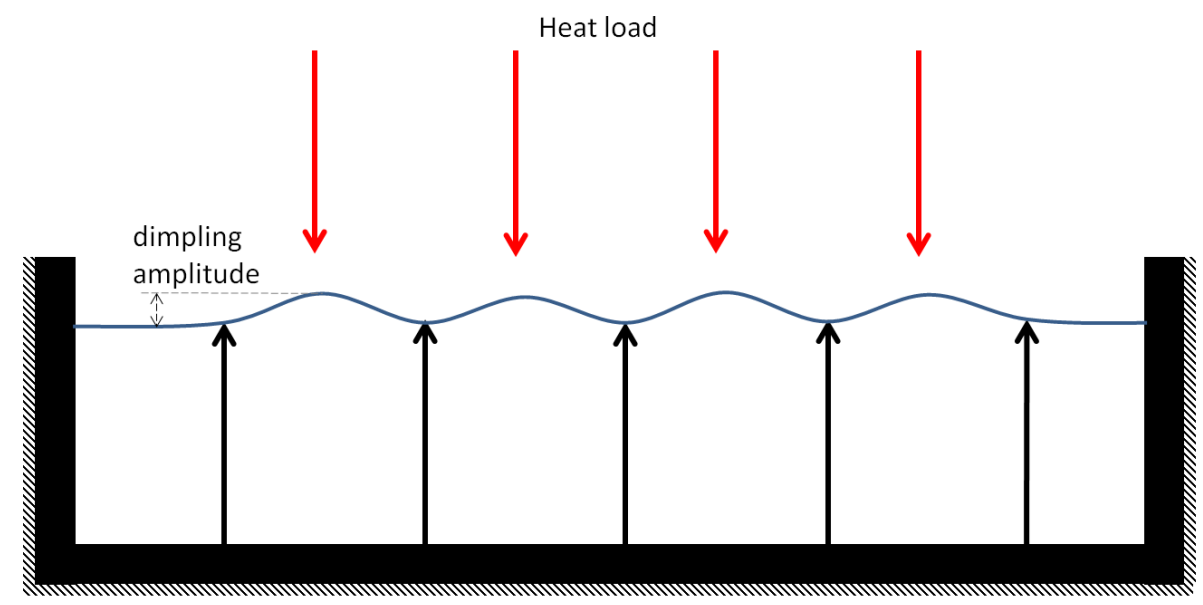

Figure 7. Schematic representation of dimpling. A heat load (i.e. an incoming high power beam) on the mirror may lead to local (periodic) deformations of the facesheet. 
Due to its inherent periodicity, dimpling will impose a two-dimensional grating structure onto the mirror surface. Such a grating will effectively generate 'copies' of the intended MP spot, each copy being a diffraction order of the dimplinginduced grating. When these orders overlap, interference between them can lead to strong modulations of the MP irradiance. The impact on the optical performance thus depends on both the amplitude (i.e. strength of the 'copies') and the pitch (i.e. amount of overlap between 'copies') of this uncorrectable two-dimensional grating.

Figure 8 shows the impact of increasing amount of dimpling-amplitude onto the RSS metric for various actuator pitches. Figure 8 provides important insights for the concept trade-off and detailed design of the HPAM module. For example, if the increase in $R S S$ due to dimpling is to be maintained within 5\%, the dimpling amplitude must be smaller than $350 \mathrm{~nm}$ for a system with $6 \mathrm{~mm}$ actuator pitch.

From Figure 8 can be learned that one strategy to deal with dimpling is to carefully minimize the amplitude of the dimples in the (thermal) design of the mirror, yet that another strategy is to make the mirror by-design immune to dimpling by ensuring that the dimpling-related diffraction orders do not overlap with (i.e. fall outside) the MP spot.

Given the $140 \mathrm{~mm}$ focal length, and the $300 \mu \mathrm{m}$ spot size, it can be easily found that in order to achieve such 'immunityby-design' the first order diffraction angle should be $>2.1 \mathrm{mrad}$ (i.e. $300 \mu \mathrm{m} / 140 \mathrm{~mm}$ ). Using the well-known grating equation, for this diffraction angle can be found:

$$
\sin \theta=m \lambda / d
$$

When it is required that the first diffracted order $(m=1)$ has a minimum angle $\theta=2.1 \mathrm{mrad}$, and considering a wavelength $\lambda=10.6 \mu \mathrm{m}$, using Equation 3 a maximum acceptable effective grating period $d=5 \mathrm{~mm}$ is found. For a rectangular actuator grid (with pitch $P$ ), the two-dimensional dimpling grating will have the largest period along its diagonals (with $d=\sqrt{2} \cdot P$ ). It is thus expected that at actuator pitches $<3.5 \mathrm{~mm}$ the system is immune to spot deformation due to dimpling. This is confirmed by the data in Figure 8.

Finally we would like to point out that when the dimpling-induced diffraction orders are outside the MP spot, obviously some light will be lost. However, for dimpling amplitudes of $200 \mathrm{~nm}$ this will result in (much) less than $1 \%$ light loss.

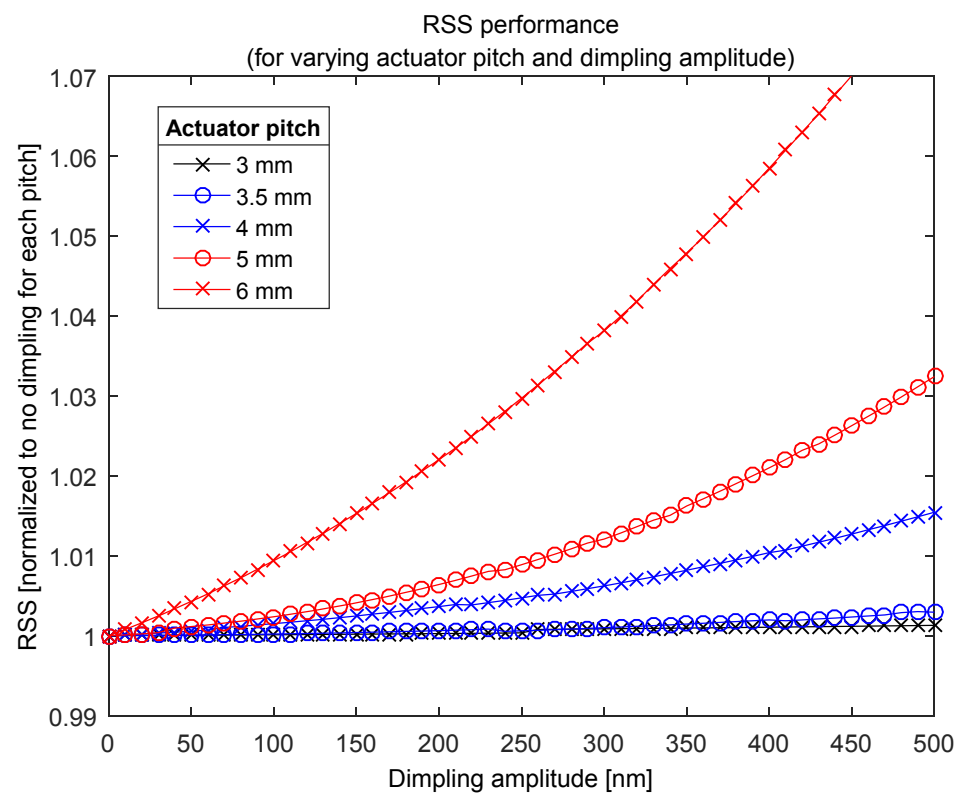

Figure 8. Relation between dimpling amplitude and optical performance (RSS). The different color curves represent systems with different pitches. Note that for clarity the vertical axis has been normalized to the $R S S$ performance in the absence of dimpling. 


\subsection{Actuator noise}

An excess amount of actuator noise can adversely affect the performance of the deformable mirror. Figure 9 shows the MP spot profiles for a system with $4 \mathrm{~mm}$ actuator pitch for varying levels of actuator noise, and Figure 10 shows the associated $R S S, U_{25 \%}$ and $U_{50 \%}$ performance. Noise has been modelled as a random (normally distributed) deviation of the intended actuator displacements.

In order to produce the best possible top hat this system requires a root-mean-square (rms) actuator displacement of $\sim 700 \mathrm{~nm}$, a maximum absolute actuator displacement of $\sim 3 \mu \mathrm{m}$, and a maximum inter-actuator stroke of $\sim 2 \mu \mathrm{m}$.

As can be seen from Figure 10 if the increase in RSS due to actuator noise is to be maintained within (for example) $5 \%$, it is required that the actuator noise must be limited to $45 \mathrm{~nm}$ rms. Figure 10 also once again clearly demonstrates the dependence of design parameters on the choice of metric. For $U_{25 \%}$ the rms actuator noise also needs to be limited to 45 $\mathrm{nm}$ for $5 \%$ performance loss, whereas for $U_{50 \%}$ more than $100 \mathrm{~nm}$ noise is allowed.

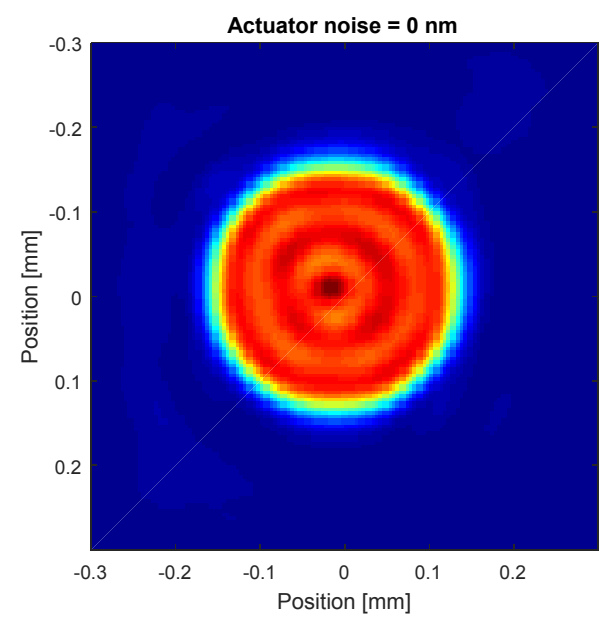

(a)

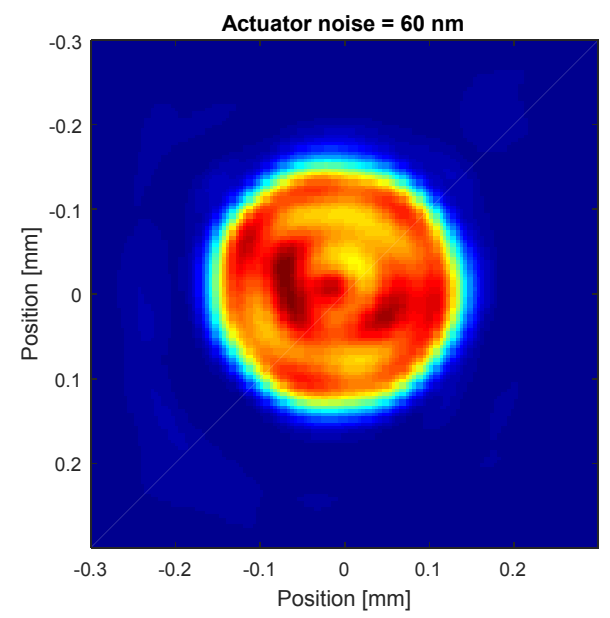

(c)

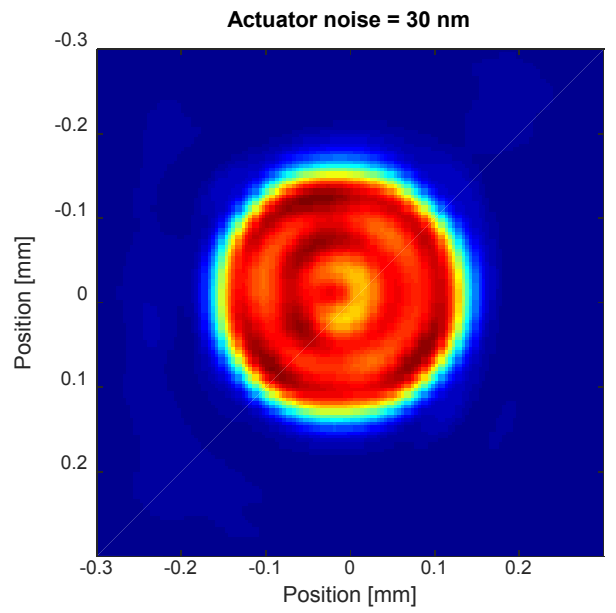

(b)

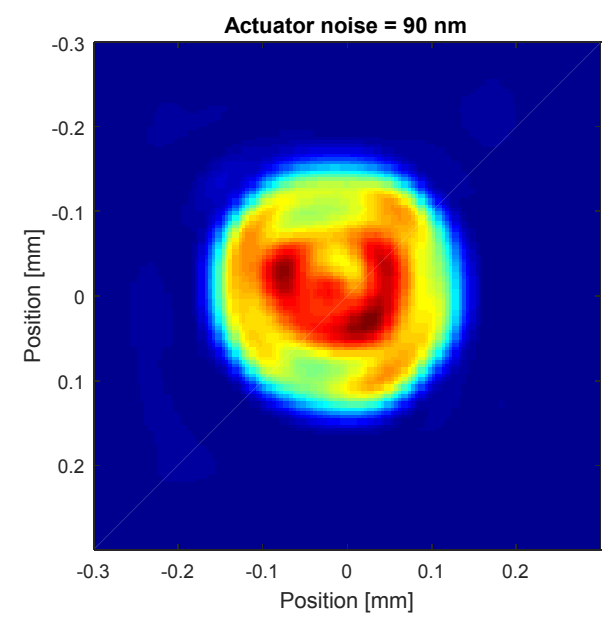

(d)

Figure 9. MP spot profile for various amounts of rms actuator noise. 


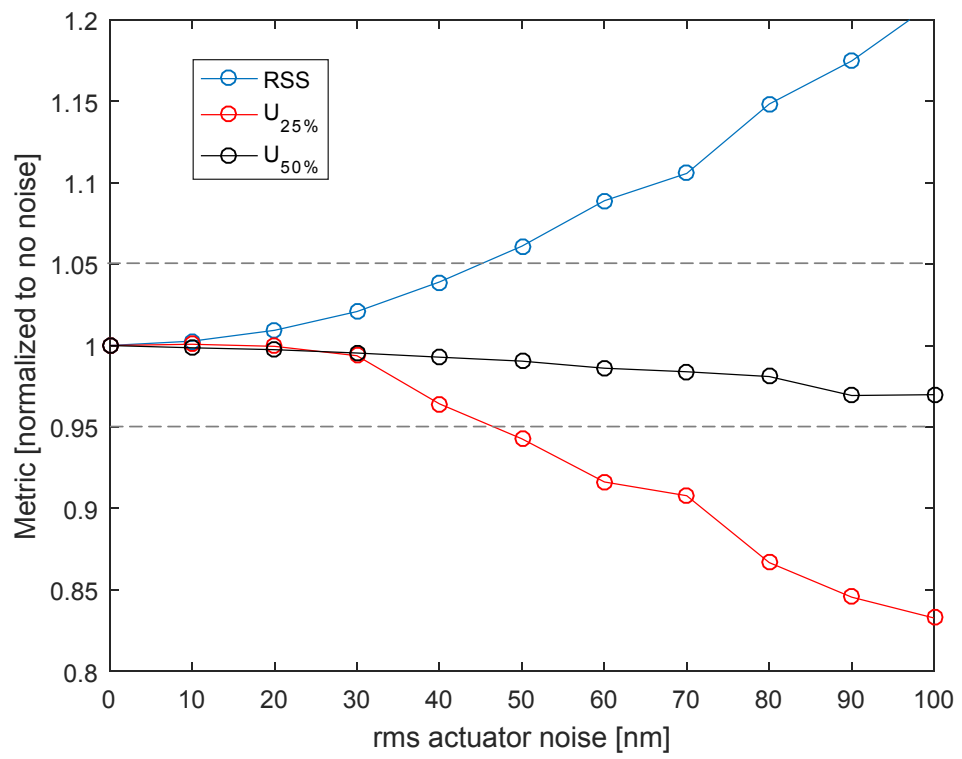

Figure 10. Relation between rms actuator noise and optical performance (RSS, $U_{25 \%}$ and $\left.U_{50 \%}\right)$. The vertical axis has been normalized to the performance in the absence of actuator noise.

\subsection{Influence function}

The design of the facesheet and choice of actuator type will affect the influence functions of the deformable mirror. The final design consideration that we would like to discuss in this paper is therefore that of the shape (and thus spatial frequency content) of the influence function.

In this paper we will not disclose in detail the requirements that we have set on the spatial frequency content of the HPAM module, but would like to introduce and explain this important issue.

When driving the actuator array with a certain actuation pattern, the resulting surface shape can be considered the convolution of a comb-array (representing the individual actuators' strokes) with the influence function (note that in practice each actuator actually may have a slightly different influence function, but this will be ignored for the sake of argument).

Since a convolution in the spatial domain corresponds to a multiplication in the frequency domain, it is clear that the deformable mirror can only produce spatial frequency content at those frequencies which are present in both the actuator grid and the influence function.

Figure 11 shows the spatial frequency content of the influence function that was measured on a deformable mirror from a third-party supplier. The dashed black line in Figure 11 indicates the Nyquist frequency corresponding to the actuator pitch, i.e. above this frequency no signal can be generated in a controlled way. Below the Nyquist frequency is the controllable area, but the influence function effectively only allows to generate frequency content at the red parts. The figure thus clearly shows that there are certain frequencies that one would expect to be able to produce (given the actuator pitch), but that cannot be practically realized. Attempting to generate those spatial frequencies anyways may result in poor performance of the deformable mirror, or would require an extreme large dynamic range of the actuator forces.

Our simulation tools allow us to assess the impact of a certain influence function throughout the design process, or compare the performance of different deformable mirrors for a given use case. 


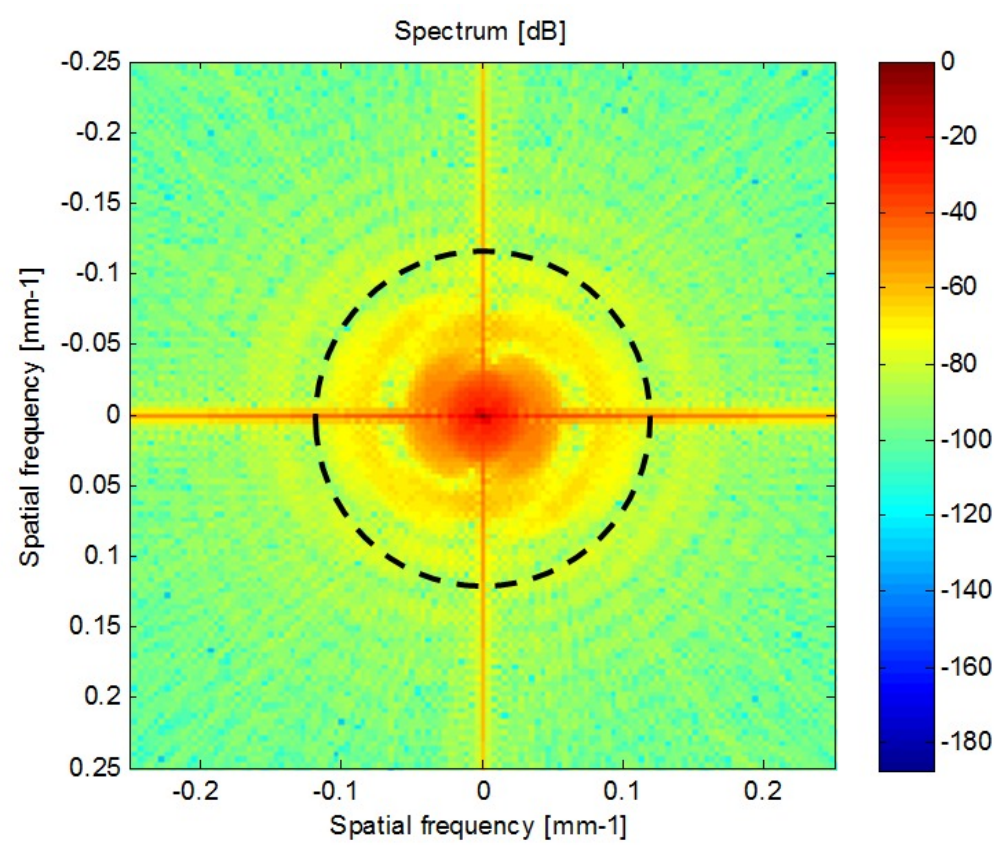

Figure 11. Experimentally determined spatial frequency content of the influence function of a deformable mirror. The dashed black line indicates the Nyquist frequency corresponding to the actuator pitch.

\section{CONCLUSIONS AND FUTURE WORK}

In this study we have developed a methodology, and the necessary simulation tools, to assess the performance and associated sensitivities of a deformable mirror to be used in the high-power $\mathrm{CO}_{2}$ laser beam path of an EUV source. TNO is currently developing such a High-Power Adaptive Mirror (HPAM).

Our analyses show that, given the current limited knowledge concerning the process window of EUV generation, the HPAM module should have an actuator pitch of $\leq 4 \mathrm{~mm}$ (if the actuator-pitch-induced $R S S$ performance impact is required to be smaller than $5 \%$ ).

Furthermore we have modelled the sensitivity of performance with respect to dimpling and actuator noise. For example, for a deformable mirror with an actuator pitch of $6 \mathrm{~mm}$, and if the associated $R S S$ performance impact is to be limited to smaller than $5 \%$, the dimpling amplitude should be less than $350 \mathrm{~nm}$. In another example, for a deformable mirror with an actuator pitch of $4 \mathrm{~mm}$, and if the associated $R S S$ performance impact is again to be limited to smaller than $5 \%$, the rms actuator noise should be smaller than $45 \mathrm{~nm}$.

Our tools assist in the detailed design process by assessing the performance impact of various design choices, including for example those that affect the shape and spectral content of the influence function.

The outcome of our work is not limited to assisting the detailed design process, after all, the optimization and wavefront propagation tools that have been developed throughout this study will be of valuable practical significance when implementing the HPAM module in an actual EUV source.

At the time of writing this paper the design process is steadily progressing. Although the design will be discussed in detail in a separate future paper, we have included some basic information for reference. Figure 12(a) shows a recent detailed drawing of the HPAM module, and Figure 12(b) shows a sample that has been manufactured to test certain critical manufacturing steps of the water-cooled facesheet. 


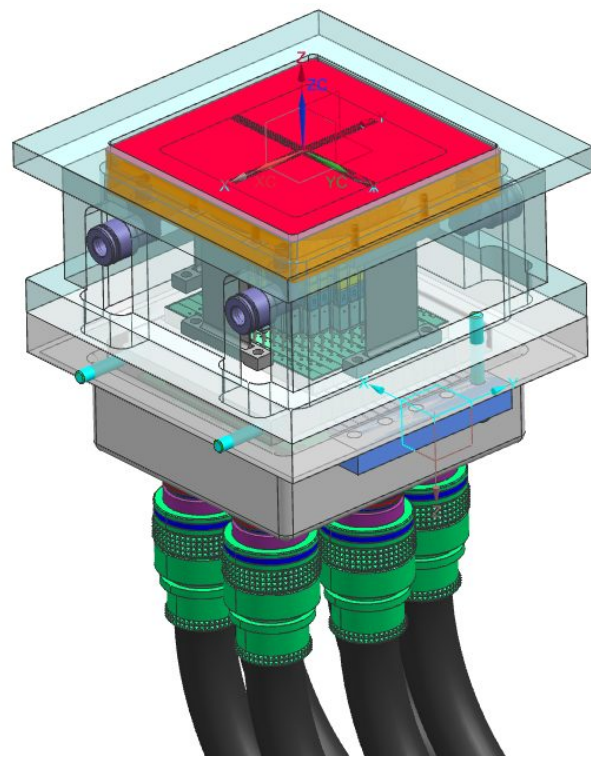

(a)

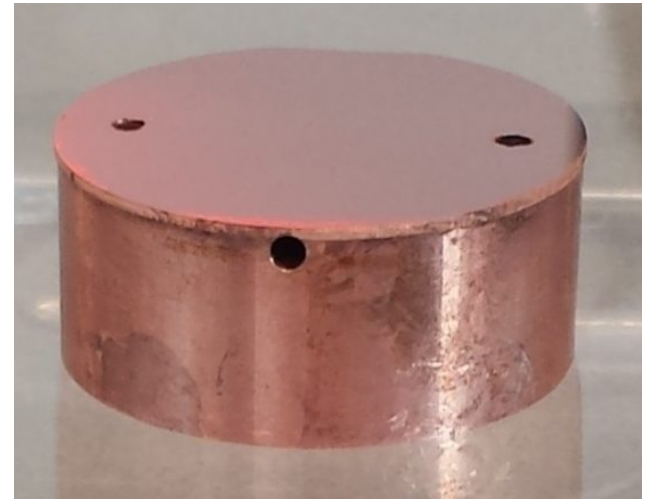

(b)

Figure 12. (a) Detailed drawing of the HPAM module, (b) sample of the water-cooled facesheet to test certain critical manufacturing steps.

\section{REFERENCES}

[1] Moore, Gordon. "Cramming More Components onto Integrated Circuits," Electronics Magazine Vol. 38, No. 8 (April 19, 1965).

[2] A. Mallik et al., "The need for EUV lithography at advanced technology for sustainable wafer cost ", Proc. SPIE 8679, Extreme Ultraviolet (EUV) Lithography IV, 86792Y (April 8, 2013).

[3] V. Bakshi, "EUV Sources for Lithography," SPIE Press (2006).

[4] H. Mizoguchi et al., "Performance of new high-power HVM LPP-EUV source ", Proc. SPIE 9776, Extreme Ultraviolet (EUV) Lithography VII, 97760J (March 18, 2016).

[5] A. A. Schafgans et al., "Performance optimization of MOPA pre-pulse LPP light source," SPIE 9422, Extreme Ultraviolet (EUV) Lithography VI, 94220B (16 March 2015).

[6] A. S. Grushin et al., "RZLINE code modeling of Sn laser-produced plasma sources of EUV radiation," Proc. Int. Workshop on EUV and Soft X-Ray Sources, paper S45, (2013).

[7] I. V. Fomenkov et al.,"Laser-Produced Plasma Light Source for EUVL," Proc. SPIE 7271, Alternative Lithographic Technologies, 727138-1 (2009).

[8] White, Dunne, and O'Sullivan, "Steady-State and Time-Dependent LPP Modeling," Lithography, Michael Wang (Ed.), InTech, DOI: 10.5772/8177 (2010).

[9] G. O'Sullivan et al., "Plasma Source Modelling for Future Lithography at $6.7 \mathrm{~nm}$ and Other Applications," Proc. Int. Workshop on EUV and Soft X-Ray Sources, paper S18, (2011).

[10] P.-Y. Madec, "Overview of deformable mirror technologies for adaptive optics and astronomy," Proc. SPIE 8447, Adaptive Optics Systems III, 844705 (September 13, 2012).

[11] J. W. Goodman, "Introduction to Fourier Optics," McGraw-Hill, New York (1968).

[12] J. W. Hardy, "Active Optics: A New Technology for the Control of Light," Proc. IEEE Vol. 66 No. 6 (1978).

[13] T. Gray and D. W. Miller, "Minimizing High Spatial Frequency Residual in Active Space Telescope Mirrors," MIT thesis (2008).

[14]R. Bastaits et al., "Segmented bimorph mirrors for adaptive optics: segment design and experiment," Applied Optics 53, pp 6635-6642 (2014). 